\title{
Research on hydrogen fuel application (in aircraft) based on carbon neutralization
}

\author{
Yan Zhang ${ }^{1, *}$, and Liang Sun ${ }^{2}$ \\ ${ }^{1}$ Economy and Management Department, Civil Aviation Management Institute of China, Beijing \\ 100102, China \\ ${ }^{2}$ Office of Academic Research, Civil Aviation Management Institute of China, Beijing 100102, China
}

\begin{abstract}
Hydrogen fuel has the advantages of green environmental protection and high energy density, hydrogen aviation is also developing rapidly in China. Due to the high-quality characteristics of hydrogen, hydrogen aviation is the key to zero pollutant emission and sustainable development of aviation industry in the future. In the context of "carbon neutralization", the EU and other countries have launched the promotion of large-scale green hydrogen plan focusing on the aviation industry. Although they are still facing some technical bottlenecks to break through some problems of hydrogen fuel in their development, hydrogen fuel will be an important means to achieve low-carbon and environmental protection in the post epidemic era.
\end{abstract}

Keywords: Hydrogen fuel; Hydrogen powered aircraft; Carbon neutralization; Challenges and suggestions.

\section{Introduction}

Under the background of realizing "carbon neutralization" to deal with global climate change, the global carbon neutralization action is accelerating the tide of green and lowcarbon transformation of the world environmental protection. Sweden's carbon peak in 1970 was 92.29 million tons, and the United States' carbon peak in 2005 reached 6130.55 million tons. By 2019, 46 countries and regions in the world had reached the peak, mainly developed countries.

There are three possible means to reduce emissions in the aviation field: improving energy efficiency, carbon capture technology and the use of alternative energy. According to the prediction of ICAO, simply improving energy efficiency can not achieve the goal of carbon neutralization, and finding alternative energy is an inevitable choice in the long term. Due to the high energy supply requirements of aircraft flight and the inability to charge midway, the battery at this stage can not solve this problem, and hydrogen fuel has high calorific value, fast hydrogenation speed and high mass power density, or becomes the key to solve this problem. At present, the application of hydrogen energy in aviation is still in the experimental stage all over the world. Although the United States, Europe, Russia and other countries have carried out relevant research and flight test, wide range application is

\footnotetext{
*Corresponding author: zhangyan1027108@126.com
} 
still a long-term goal. As the world's largest aircraft manufacturer, Airbus takes hydrogen fuel as the most promising energy type and renewable energy to provide power for aircraft and aviation. In 2020, it announced three hydrogen energy concept aircraft, becoming the world's first zero emission aircraft, code named zeroe, which will change the future air transportation mode and be launched in 2035 .

Under the background that China has achieved the goal of "carbon neutralization" in 2060, hydrogen energy as a secondary energy is indispensable. Due to the limited options for emission reduction in the field of civil aviation, hydrogen fuel as a way to reduce carbon emissions will become the key strategy and long-term key path of the net zero goal. In 2019, the North research center of COMAC and the hydrogen energy technology development company of State Power Investment Group launched the development of "lingque H" new energy verification machine, which was successfully tested at Zhengzhou Shangjie airport. In March 2019, Dalian Institute of chemistry, Chinese Academy of Sciences and Liaoning General Aviation Research Institute jointly developed the first manned hydrogen fuel cell test machine, which successfully flew in Shenyang ${ }^{[1-2]}$.

After exploration in the past few years, all countries urgently hope to accelerate the application of hydrogen energy in aviation, especially in the wide range field of aviation.

\section{Characteristics of hydrogen fuel}

Hydrogen, with the element symbol $\mathrm{H}$, is the lightest chemical element. It is a gas at normal temperature and pressure. As the most common element, it is mainly stored in water. Since NASA technicians successfully used hydrogen as fuel for the Saturn V lunar rocket, fuel experts are more convinced that hydrogen can be used as an ideal fuel to replace coal, natural gas and oil, and believe that mass production of hydrogen can be achieved, even in atomic power stations. Hydrogen is considered as one of the major energy solutions of the twenty-first century, capable of meeting future energy needs. Hydrogen is clean, sustainable fuel and it has promise as a future energy carrier. It also has the ability to substitute the present energy infrastructure which is based on fossil fuel. Being 61a zeroemission fuel, it could reduce environmental impacts and craft novel energy opportunities. ${ }^{[3-4]}$

Among the renewable sources that are considered to produce hydrogen, wind and solar energies are most commonly used for hydrogen production up to date. Most of the methods for the production of green hydrogen consider obtaining hydrogen either through water splitting reactions (e.g. water electrolysis, water thermolysis, photocatalytic water splitting, and thermochemical water splitting) or via dehydrogenation of hydrogen carrier molecules. ${ }^{[5]}$ The chemical energy of hydrogen can be converted into thermal energy or electric energy, so one of the wide applications of hydrogen is as aerospace fuel. The different characteristics of hydrogen determine that hydrogen has different application technologies [6].

\subsection{Buoyancy of hydrogen}

As hydrogen is the lightest of all gases, its density is only $0.0899 \mathrm{~kg} / \mathrm{m}^{3}$ at $0{ }^{\circ} \mathrm{C}$ and its density is small, so hydrogen has the largest buoyancy and can be used as lifting power.

\subsection{High energy properties of liquid hydrogen}

In any chemical fuel, the specific energy (unit mass energy) of hydrogen is the largest, and the low calorific value is as high as $120 \mathrm{mj} / \mathrm{kg}$, which is about 3 times that of gasoline and 
kerosene and 4.5 times that of carbon. The power and range of aeroengine are directly proportional to the calorific value of fuel. Using liquid hydrogen as aviation fuel can greatly improve all performance parameters of aircraft, and the takeoff weight of supersonic aircraft fueled with liquid hydrogen is only half of that of kerosene. Lockheed has conducted comparative experiments on aviation kerosene and liquid hydrogen. It is found that under the same payload and range, liquid hydrogen fuel is much lighter, the total weight of the aircraft can be reduced, and the running time can be shortened, thus saving the total fuel consumption.

\subsection{Environmental emission characteristics of hydrogen}

As a carbon free fuel, hydrogen does not produce $\mathrm{CO}, \mathrm{CO}_{2}$ and $\mathrm{CH}$ compounds emitted during hydrocarbon combustion, and will not produce greenhouse effect. Hydrogen is a colorless, odorless, odorless and non-toxic clean fuel. Burning in pure oxygen only produces water, which returns to nature and maintains ecological balance. Advanced hydrogen fuel cell and catalytic hydrogen technology only discharge water. Using these hydrogen energy to drive vehicles and aircraft can achieve real "zero emission". The United States has held the vehicle competition with the least air pollution, and it is concluded that the hydrogen engine won the first place, and the exhaust gas is even cleaner than the inhaled urban air.

\subsection{Hydrogen fuel resources}

As an important characteristic of new energy, it is rich in resources and wide sources. The earth's atmosphere contains 1.6 billion tons of hydrogen, but free hydrogen is difficult to use. At present, more than $90 \%$ of the world's hydrogen fuel is produced by fossil fuels, and part of it is produced by electrolysis. With the development and breakthrough of hydrogen energy technology, electrolytic water and dissociated steam will be gradually transformed, and the storage capacity of water in Shanghai is $1.3 \times 10^{18}$ tons, water can be recycled. Therefore, hydrogen energy is not only inexhaustible, but also inexhaustible. According to the investigation, there are 1 billion tons of water on the moon, and Jupiter is 318 times that of the earth. It is a large natural warehouse of hydrogen resources, which will be developed and utilized by mankind one day.

\section{Challenges of hydrogen fuel application in Civil Aviation}

Since the invention of aircraft in the early 20th century, mankind has not stopped the exploration and application of new technologies. However, hydrogen energy has attracted more and more attention because of its superior properties. Therefore, although the application of hydrogen energy to aircraft has a series of obvious advantages in theory, there are still many problems to be solved if it is really put into wide range application.

\subsection{Production, storage and use of hydrogen fuel}

Compared with lithium battery pack as battery system, hydrogen fuel can prolong the service life by 10 times and increase the energy density by 5-20 times. Hydrogen energy has unique application prospects. However, over the past few decades, the aviation industry has had a comprehensive understanding of aviation kerosene used by turbine and turbofan engines and accumulated rich experience in safe storage, transportation and use of these system fuels. The production, storage and safe and effective use of hydrogen fuel need the 
joint cooperation of government regulators and manufacturers to achieve innovative breakthroughs in management measures and key technologies. At present, there are still many unknown fields in the related theory, knowledge and application of hydrogen energy [7].

\subsection{Infrastructure and supporting facilities}

Supporting the propulsion system of hydrogen powered aircraft requires new ground processes, such as filling hydrogen fuel in the shortest possible time to make the parking time of the aircraft as short as possible. How to ensure the safety of these processes.

The operation of hydrogen powered aircraft must be determined at an early stage and take into account the construction of infrastructure. At present, both airlines and airport supporting facilities are prepared for aviation kerosene. Therefore, the main difficulties in promoting hydrogen energy include the lack of infrastructure.

\subsection{Policy support}

To achieve the goal of "carbon neutralization" and zero carbon dioxide emission as soon as possible, we need not only the support of technology research and development, but also the support of policies.

In the United States, $85 \%$ of flights are concentrated in 40 airports, and the remaining $15 \%$ are distributed in more than 450 airports. The larger the energy filling network, the more difficult it is to replace it as a whole, and the higher the cost. Each smaller station needs a more complex fuel logistics support system, and each kilogram of fuel allocated will produce a higher amortization cost. At the same time, The overall upgrading of the fuel system requires a lot of capital and human investment. It is difficult to imagine without the support of local governments.

\section{Enlightenment and suggestions of hydrogen aircraft}

At present, according to the research on hydrogen powered aircraft by major research institutions and enterprises and the judgment of relevant development trends, the following enlightenment is given to the technological development of aviation "carbon neutralization" in China as soon as possible ${ }^{[8]}$.

\subsection{Develop air route map}

Facing the challenge and urgency of decarbonization in the aviation industry, it is very necessary to formulate a systematic strategy and a clear aviation roadmap. It is difficult to put hydrogen powered aircraft into use in the short term and faces great challenges. From the perspective of China's current development, the aviation industry is very key and promising in realizing carbon neutralization. Vigorously developing hydrogen aviation technology is not only very conducive to achieving emission reduction targets and promoting the early realization of carbon neutralization, but also driving the systematic innovation and improvement of aviation technology. In order to achieve the goal of Green Aviation in China as soon as possible, it is suggested to formulate the corresponding road map as soon as possible and speed up the performance of relevant technologies.

\subsection{Accelerate the exploration and research of hydrogen powered aviation technology}


The application potential of hydrogen propulsion technology is encouraging. Research and innovation are very important to realize the potential of hydrogen technology in aviation decarbonization. Europe, America and other countries have carried out various research on hydrogen aircraft technology. China has also carried out relevant research and is in a leading position in some fields. In order to continue to play our role, it is recommended that government departments strengthen organization, systematically plan and study the design technology, fuel supply chain technology and infrastructure development of hydrogen energy to promote aircraft, scientifically plan the application of hydrogen energy in aviation industry and break through various key technologies.

\subsection{Speed up the exploration and research of new aircraft aerodynamic layout}

In order to give full play to the advantages of new energy propulsion technology, innovative aerodynamic layout research is carried out for the effective integration of airframe and propulsion system to improve the overall efficiency of the aircraft. At present, there is still a certain gap between the relevant research carried out in China and the engineering application in terms of breadth, depth and systematicness. The government departments should strengthen the organization, and the research institutions can carry out systematic planning research on the introduction technologies such as new start-up layout, and pay attention to the sustainability and inheritance of the research results.

\section{Conclusion}

Although the production cost of hydrogen is high, there is no complete hydrogen transmission pipe network, high transportation cost and safety, there are still many difficulties to be overcome, but the development prospect of hydrogen fuel cell is good. With the decarbonization of aviation industry and the support of various countries for the development of hydrogen energy, the research of hydrogen fuel in aircraft in aviation industry is worth looking forward to. Through the planning and formulation of aviation roadmap, accelerating the exploration of hydrogen powered aviation technology, the exploration of new aircraft aerodynamic layout and aircraft life cycle assessment research, hydrogen fuel will become an important path to achieve low-carbon and environmental protection in the future.

\section{References}

1. Min Sun, Large aircraft 9, 45 (2020)

2. Yangjun Zhang, etal, Aerospace Power 1,20(2021)

3. Manish Kumar Singla, etal, Environmental Science and Pollution Research 28, 1560(2021)

4. Shashi Sharma, etal, Energies 14, 7389(2021)

5. SelmaAtilhan, etal, Current Opinion in Chemical Engineering 31, 100668(2021)

6. Zhengju Chen, Deping Wang, Journal of Shenyang Institute of Aeronautical Engineering 27, 88 (1994)

7. Guoxiong Dong, Large aircraft 10, 24 (2020)

8. Miaoxiang Wang, Aerospace Power 1, 16 (2021) 